\title{
Indexicals in Virtual Environments
}

\author{
Bernardo Alonso \\ Department of Philosophy, Universidade Federal de Mato Grosso, Cuiabá, Brazil \\ Email: bernardoalonso@ufmt.br
}

Received 10 February 2014; revised 10 March 2014; accepted 17 March 2014

Copyright () 2014 by author and Scientific Research Publishing Inc.

This work is licensed under the Creative Commons Attribution International License (CC BY).

http://creativecommons.org/licenses/by/4.0/

(c) (i)

\begin{abstract}
In this paper I explored three well-known cases that seem to cast doubt on the notion that a speaker is always at the place of the utterance when the utterance occurs. I gave a few examples produced in Second Life environment, which cannot be handled correctly by evaluating the expression at issue with respect to the traditional view, i.e., the kaplanian framework-where the agent and the utterer will always be identical, and the referent of "I" will always be the utterer. The same happens to "here" and "now"; in each case the character of the indexical is a function from a contextual parameter to the referent of the expression. An example of logical truth peculiar to indexicals is the sentence "I am here now", since, according to that view, no utterance can take place in a context whose agent is not in its location in its time. Finally I concluded by claiming that particular kinds of utterances produced in virtual environments (VE) break with the logical truth status of "I am here now".
\end{abstract}

\section{Keywords}

Indexicals; Kaplan; Virtual Environments; Context; Utterance

\section{Introduction}

This study of indexicals not only can shed light on general theories of meaning, but also can give some insight into matters such as belief, knowledge, first-person perspective, personal identity and consciousness ${ }^{1}$. The expression "index" was introduced by Charles Sanders Peirce (1935: 2.274-2.308, in Brinck, 1997). He maintained that an indexical was characterized by standing in a contiguous relation in time and space to which it indicated. It depends on the existence of its object to function as a sign. Peirce holds that demonstratives, pronouns and proper names are indices. According to him, linguistic indexicals focus the attention of the hearer on an object common to the experience of both speaker and hearer. Thus the indexicals establish a "real connection" between the hearer and object, and they do so without describing the object. Kaplan does not include proper names ${ }^{1}$ I would like to thank the patience, availability and critics of a few professors, in special Prof. Luciano Floridi, Peter Ludlow, Marco Ruffino and André Fuhrman. 
in the class of indexicals. As opposed to proper names, indexicals do not hold on their objects over time. Rather, their reference is momentary, tied up with the instance of use. "I" uttered by $a$ no longer indicates $a$ when uttered by $b$. "Now" uttered at $t 1$ does not still indicate $t 1$ at $t 2$ and "here" uttered at $l 1$ does not still indicate $l 1$ at $l 2$.

In the kaplanian framework (1989) the indexical's referent and content are determined by its linguistic meaning and contextual factors. Each context $c$ has at least an agent, time, location, and possible world associated with it $-<a, t, l, w, \ldots>$. The component of meaning that he calls "content" is a function from possible worlds to referents, or in a kaplanian vocabulary, a function from circumstances of evaluation to an appropriate extension. The content of a given utterance of a sentence is the proposition expressed, but parts of sentences (names, predicates, indexicals, etc.) also have contents. The evaluation of the content of an expression in a given circumstance yields its extension in that circumstance (1989: p. 501). Hence, the evaluation of a proposition yields a truth-value, the evaluation of a singular term yields an object and the evaluation of an n-place predicate is a set of ordered n-tuples.

The "character" of an expression is a function from possible contexts of use to contents. It is what "determines the content in varying contexts" (1989: p. 505), e.g. the character of "I" can be represented as a function, which takes as input the contextual parameter, the agent, and returns as value the referent. The "character" aims to capture the linguistic meaning of indexical expressions.

The reference and content of a true demonstrative (e.g. "that") in a context depends on the speaker's intentions like the one manifested by a pointing gesture. But the reference and content of a pure indexical is "automatic" (Perry, 2001), they do not depend on the speaker's intentions. The content of "I" with respect to a context $c$ is the agent of $c$; the content of "here" is the location of $c$; the content of "now" is the time of $c$.

Kaplan's logic of demonstratives (LD) involves indexicals, demonstratives, tense operators, contexts of utterance, agents, positions, times, and the distinction between character and content, but despite the requirement of a much more powerful logical apparatus than simple [quantified] modal logic (Zalta, 1988), Kaplan argues for two "obvious" principles (Kaplan, 1989: p. 492):

1) The referent of a pure indexical depends on the context, and the referent of a demonstrative depends on the associated demonstration.

2) Indexicals, pure and demonstrative alike, are directly referential (That is, the content an indexical yields is its denotation, not a sense or other conceptual component).

Kaplan argues that as utterances are features of the world and hence occur in time, it is not possible to provide a semantic evaluation of utterances. We need instead to set the abstract notion of a sentence-in-a-context, and to do this we need the notion of a context. Considering that a context is a "possible occasion of use" (1989: p. 494) of an expression, the agent turns out to be an abstract feature of the semantic framework, a contextual parameter filling the argument of the character, giving us the referent of the indexical, and, as such, is logically distinct from the notion of an utterer. But in the kaplanian framework the agent and the utterer will always be identical and the referent of "I" will always be the utterer. The same happens to "here" and "now"; in each case the character of the indexical is a function from a contextual parameter to the referent of the expression.

The logic of demonstratives (LD) states that a sentence is valid, and is a logical truth, if it is true at every context in every LD structure. An example of logical truth peculiar to indexicals is the sentence "I am here now", since no utterance can take place in a context whose agent is not in its location in its time. In this paper I will argue that an appropriate semantics should not classify "I am here now" as a logical truth. In order to do so I will present examples of false utterances of "I am here now" and true utterances of "I am not here now". Such examples will differ from the ones present in the well-known answering machine and post-it puzzles (Sidelle, 1991; Predelli, 1998a, 1998b; Corazza et al., 2002).

In the next section I sketch some of the examples found in the literature that challenges the traditional view ${ }^{2}$. In Section 2 I give some examples produced in Second Life environment, which cannot be handled correctly by evaluating the expression at issue with respect to the traditional view. I conclude this paper by claiming that utterances produced in virtual environments (VE) break with the logical truth status of "I am here now" sentences.

\section{Well-Known Puzzles}

“One needs only understand the meaning of ['I am here now'] to know that it cannot be uttered falsely”.(Kaplan,

${ }^{2}$ Similar to what Stefano Predelli calls Simple-Minded View- “(...) the claim that utterances (or inscriptions) are always correctly represented by means of indexes whose co-ordinates correspond in an obvious manner to the parameters of the context of utterance (or inscription)" (Predelli, 2005: pp. 41-42). In a rough manner, the referent of "I" is ALWAYS the agent of the utterance, the referent of "here" is ALWAYS the location of the utterance and the referent of "now" is ALWAYS the time of the utterance. 
1989: p. 509).

There are two well-known puzzles that criticize the kaplanian framework for the indexes "now" and "here" (Sidelle, 1991; Predelli, 1998a, 2005) and a case where it's claimed that "I" does not always refer to the agent of the context, among other variants ${ }^{3}$. The first two are metasemantic or pre-semantic (Perry, 1997; Dodd \& Sweeney, 2009) claims. They involve finding the suitable condition from where you can start doing semantics, e.g. questions about in virtue of what does a given term have its semantic value (metasemantic) and about suitable associations between uttered sentences and contexts (pre-semantic). The latter is rather a semantic claim, since it's about the character of "I", as we'll see later.

Sidelle's paper makes the very simple point that utterances of "I am here now" do not always seem to be true in the trivial way that Kaplan had assumed. In particular, when you call somebody with an answering machine it is possible to hear his voice saying, "I am not here now". In this case we seem to have the strong intuition that he is telling the truth if he really isn't at home. If "I am not here now" can be uttered truly, we need to allow contexts $c^{*}$ (class of improper contexts) ${ }^{4}$ into semantics such that the semantics will take <"I am not here now", $c^{*}>$ to a true proposition. When such contexts are paired with "I am here now" the semantics will take the pair to a contradiction, and thus "I am here now" will not be a logical truth.

According to a footnote in the Demonstratives, where Kaplan attributes to Keith Donnellan, "our language might contain two forms of 'now': one for the time of production, another for the time of audition" (Kaplan, 1989: p. 491, fn. 12). That seems to be how Sidelle deals with the Kaplan's dictum that the referent of "now" is the time of the utterance, i.e. the utterance of an answering machine message occurs when the person records the message. In fact Sidelle solves the puzzle arguing that the utterance takes place when the message is heard by the listener, the decoding time, not when it is recorded, the encoding time (Fillmore 1975, in Predelli, 2005). The kaplanian framework is left untouched—at the time the answering machine answers a call by reproducing the message "I am not here now" a truth is expressed and the characters of the indexicals remain the same. The referent of "now" is the time the call is made (the triggering of the answering machine, not the time of the recording). So "I" continues to refer to the agent $a$, who is not "here" ( $l$, the contextual parameter of location, the place where the machine is located) "now" (when the message is heard).

Predelli $(1998 a, 2005)$ presented a similar case using a post-it example. He tells us the story of Jones, who suddenly decides to flee the country. Before leaving home at eight in the morning, he writes a note to his wife, who will be back from work at five in the evening.

"As you can see, I am not at home now. If you hurry, you'll catch the evening flight to Los Cabos. Meet me in six hours at the Cabo Real."

The note does not convey the false message that Jones is not at home at the time the note was written. It also doe not request that his wife be at the hotel at 2 p.m. (six hours from the inscription). Predelli tells us that the correct interpretation of this example may be obtained on the basis of the character of "I", "here" and "now", and of assumptions pertaining to the interpretative system's structure, "if the index taken into consideration by the system contains coordinates intended by the speaker as semantically relevant, even if distinct from the obvious items within the context of utterance/inscription” (Predelli, 2005: p. 44). Predelli's intentionalist position is quite controversial and has been criticized in a number of articles (Corazza et al., 2002; Romdenh-Romluc, 2002; Dodd \& Sweenwy, 2009). He thinks agent’s intentions can force the hearer/reader/receiver to look at a context of interpretation, since the index yielding the appropriate outcome contains as its temporal coordinate the expected time at of Mrs Jones's arrival, 5 p.m., rather than he time at which Jones wrote the note, 8 a.m. Predelli argues that, with respect to this index, "now" and "in six hours" succeed in picking up 5 p.m. and 11 p.m. according to their customary characters. According to him, temporal indexicals are ambiguously anchored to either temporal coordinate, and the appropriate level of lexical representation ought to distinguish between the "now" of the time of production and the "now" of the time of audition. Depending on the meaning with which indexicals are used, they may end up picking up an individual a, location $l$, and time $t$, such that a may well fail to be in $l$ at $t$. Now imagine if Jones's wife is unexpectedly delayed and arrives at home only at 8 p.m. According to what Predelli calls the Simple Minded View, "now" in Jones's note should pick out the time of utterance, the time Mrs Jones reads the note (Donnellan's time of audition), but Predelli argues that Mrs Jones, being aware that she was expected home at 5 p.m., will not arrive at the hotel only at 2 a.m., but will meet her husband,

${ }^{3}$ In a number of recent papers the Kaplanian framework for the logical truth status of "I am here now" has been criticized. Trying to sketch all of them would go beyond the main objective of this paper, that is to present one simple new case.

${ }^{4}$ They would be contexts where $a$ was not at $l$ at $t$ at $w$-Kaplan did not include such contexts in his semantics. 
as Jones expects, at 11 p.m. On Predelli’s account, "now” can be sensitive to an intentional context which may well differ from the context of utterance.

The final case I had like to consider before the next section was suggested by Corazza et al., 2002 and involves the claim that "I" does not always refer to the agent of the context. Their proposal retain the account of the character of "I" as a function from agent to referent, pace Kaplan, but reject the agent-utterer identity in problem cases. Understood along the lines of something like a Wittgensteinian language game (2002: p. 11, fn. 10 ), the contextual parameter of the agent is conventionally given by the social or conventional setting in which the utterance takes place.

For instance, in the answering machine case, the context in which "now" is used changes the time that the term refers to. If a friend sent you a postcard from India saying "It's snowing now in Manali", the relevant time to be taken is the time those words were written. But in the answering machine case when "now" is heard, the relevant time to be considered is the exact time of the message being played.

Corazza's aim is to challenge the pure indexical's status of "I" in a slightly different manner than Predelli's intentionalist picture, which is claimed to be too strong - since too much is left for the intentions of the agent. Let's analyse Corazza's post-it example:

"Joe is not in his office one day and Ben notices that a number of students keep approaching his door and knocking. They then stand around and look bemused for a while before leaving. Taking pity on these poor souls wasting their time, Ben decides to attach his 'I am not here today' note to Joe's door. The trick works; the students, instead of knocking and waiting, take one look at the note and then leave” (Corrazza et al., 2002: p. 5).

In this example it is clear that "here" and "now" refer to the day and place the note is read, but in the case of "I” things get a little bit more complicated. Joe didn't write the note; neither placed it on his door. Corazza suggests that it is implausible to suppose that Joe is the utterer in this situation. If one takes into account kaplan's character of "I" ("I" refers to the agent and the agent is identical to the utterer) it seems counter-intuitive that one can make utterances without playing no role in its production. Instead of that, it is claimed that, although "I" refers to Joe, Joe is nevertheless not the utterer. Kaplan's account of the character of "I" as a function from agent to referent is retained, but the agent—utterer identity is rejected.

In a recent paper (Dodd \& Sweeney, 2009) this position is criticized ${ }^{5}$ with the support of three elements (stages) of utterance production. In STAGE 1 the agent of the context kicks the mechanism of production into action (time, location $=t 1, l 1$ ). In STAGE 2 the mechanism of production works towards its end, namely producing a sentence tokening. In STAGE 3 the sentence is tokened, and the agent's message is now broadcast to her audience (time, location $=t 2, l 2$ ). In the post-it example above, Dodd and Sweeney argue that when using the personal indexical "I", a potential agent other than the referent can only be involved as part of the mechanism of communication. When Ben places the "I am not here today" note on Joe's door he is communicating something, but it is argued that there is no agent of the context and that the proposition is left incomplete. Joe cannot be the agent since Ben acted completely on his own initiative. "I" doesn't refer in this case. In Perry's (2003) terminology, there is no referential content.

\section{Virtual Environments}

My suggestion is that in virtual environments, e.g. Second Life, one has to give up the pure indexical status of "here", but the pure indexical status of "I" is preserved even if "I" refers to an avatar. "Now" is not a worry in the virtual environments cases I present since the examples involve real time situations. The lag, the delay between a keyboard inscription (or a microphone utterance) and its tokening is regarded as a [unfortunate] constituent part, as a side effect of the mechanism of production, pace (Dodd \& Sweeney, 2009).

Here is the scenario. In Second Life you can be whoever you want (with the programming limitations in mind), you can fly, change gender, change shape, size, the colour of your skin, teleport and build your own virtual world parcel. That's great when you think of entertainment, the enormous possibilities of gaming, fictional characters development, fantasy graphics and use of creative content. But all these have to do with the eye-candy of Second Life. The virtual world is not about the stuff you actually see, the things you can build are only symbols that serve as a kind of catalyst for the formation of social groups (Ludlow, 2009). There are thousands of residents who spend long hours, sometimes too much of their time inside the Second Life environment. It's the people who work there, programmers, designers, architects, teachers, people who run business, who participate

${ }^{5}$ Dodd and Sweeney defend Kaplan's claim that "I" is a pure indexical, however they criticize the claim that "I am here now" is a logical truth as they allow "improper contexts" to be part of their semantics. 
in learning projects, meetings and don't take that environment only as a simple communication medium, but as a new geographical situ (Ondrejka, 2007). More than working towards producing sentences tokenings, virtual worlds are meant to be powerful environments that can dramatically improve collaboration and interaction between agents immersed in a huge 3D environment. This new situ overcomes distances bypassing traditional geographical constraints.

Example (1):

I met Professor Peter Ludlow in a conference at Universidade Federal do Rio do Janeiro and we chatted about Second Life. We exchanged our Second Life resident names-Urizenus Skalar (Professor Peter Ludlow) and Bardo Dejavu (me) - and made plans to meet in SL to share some thoughts about indexicals in virtual environments. Three days later I logged in from Rio at 15:35 p.m., as well as Professor Ludlow at 13:30 p.m. from New York. Since we are friends in SL we can see each other status in our friends list, hence when I log in he might be aware (if he searches) that I'm logged in and vice-versa. As I was five minutes late he was the one who received the message that I've logged in. He sent me a text message "I am here now" and offered me a teleportation to the location where he actually was. As soon as the teleportation was complete something happened at his [real $]^{6}$ house - the pizza was burning in the oven — and he had to leave for a moment to rescue his lunch, changing his status message to "away"7. Curiously Ludlow programmed his status to display "I am not here now" instead of "away". So, after the teleportation, when I finally reached the parcel of the virtual environment he was supposed to be I saw his avatar sleeping with the status message upon his head "I am not here now".

Case (1): "Here" is not a pure indexical.

Considering the traditional view-that the utterer and the agent are always the same-and considering the avatar as a fictional character, as a medium, part of the mechanism of production, when Ludlow produced "I am not here now" in SL a truth was expressed. In this case "I" refers to Professor Peter Ludlow, "now" to the exact moment that I read the message, but "here" is not the location from where he uttered the message (New York). "Here" refers to a 3D parcel inside huge servers in Texas rendered in a proper display apparatus. If I was teleported to the wrong parcel I couldn't read the message. "Here" is not a pure indexical, the contextual location at the time of the utterance is not the same as the location of the utterer.

One possible objection is to affirm that, when Ludlow left the message "I am not here now" he meant "not behind the computer" simply because he run for his pizza. This objection only reinforces that "I am here now" is not a logical truth, because, kept the purity of the indexical "here"-the referent of "here" is always the location of the utterance-he truly uttered "I am not here now" while keeping the kaplanian framework intact, but that's not what it's claimed. The example above was produced on purpose to force this objection. Instead of the burning pizza, Ludlow could simply be tired of waiting and just set his status as away ("I am not here now"), while still sat on his chair, in front of the computer screen. Would this be a false proposition? I don't think so, simply because "here" doesn't mean "in front of the computer" in this case.

It is important to notice the dual use of "here"-pure indexical (here) vs demonstrative (dthat $[\alpha]$ ). As suggested by Colterjohn and MacIntosh (1987), "here" may pick up a location distinct from where the speaker happens to be. According to their suggestion one can say: "I am here now, but tomorrow I'll be here" (pointing to Amsterdam on a map). In their view, "here" operates as a demonstrative accompanied by a "proxy finger". In our case "here" operates as a demonstrative accompanied by pixels and hyperlinks.

Example (2):

Urizenus Skalar, a famous journalist from the Second Life Herald, is going to meet Bardo Dejavu, a cyberpunk resident, for an interview about the constant raise of rental prices in SL. But when Skalar teleported to Bardo's house he noted that Bardo had already logged off and left a message on his door- "I am not here now".

Case (2): "Here" is not a pure indexical, neither is "I"?

"Here" refers to the 3D location in the SL parcel, "now" refers to the time Skalar reads the status message, but in this case it is not clear that "I" refers to me, the utterer. One can argue that in this case "I" refers to Bardo and that Bardo is more than a mere mechanism of production. In this case Bardo is the agent $a$ and the agent is different from the uterrer. He represents the "Avatar" in the strong sense of the term, a form of self, representing the electronic embodiment of the user. Note that this is not a naïve assumption. The example above is different from example (1) because Bardo is not "away"; the avatar is not "sleeping” with a message upon his head conveying the information that the gamer, the person behind the computer is not available at that moment. The

\footnotetext{
${ }^{6}$ As opposed to "virtual".

${ }^{7}$ One can set status messages to avatars, if you're “away” means you're still logged into your account but not present at the moment.
} 
message is clear; it states that the avatar is not there.

Is this case like Corazza's post-it case but in cyberspace? Is it plausible to affirm that there are cases where this kind of embodiment allows a different index, which constitutes improper contexts with respect to "I"? My answer is no.

In third person virtual environments like SL the avatar works as a rich mechanism of production, an IT augmented version of "I", a kind of costume to whatever social role you play. The richest and complex it might be, it is still "you" talking, typing, flying, logging off. If the avatar is the graphical representation of a user, the utterer-agent identity is kept, hence "I" is still a pure indexical. The word "I" has as the contextual parameter the agent, that is the utterer, who is speaking/typing at the encoding moment. It is plausible to assume that there are many cases in which a SL resident tells another resident "I am here now" he doesn't mean "at home, in front of my computer, speaking through the microphone". In these cases he obviously means "in this parcel of this virtual environment". On the other hand it is particularly implausible to assume that in these cases "I" doesn't refer, ultimately, to the person behind the computer, even if it's through a kind of alter ego or fantastic representation ${ }^{8}$.

\section{Conclusion}

The notion that a speaker is at the location of her utterance when she is producing is widely accepted. Given the notion that "I", "here" and "now" refer to the utterer, the place and the location at which the utterance takes place, it follows that sentences such as "I" am here now' are always truly utterable. We saw that there are some cases that seem to cast doubt on the notion that a speaker is always at the place of the utterance when the utterance occurs. These cases appeal to improper contexts, that is, indexes $i$ such that $i a$ is not in $i l$, at $i t$ and $i_{w}$. The first two cases concern the status of pure indexicality of "now" and "here", the third of "I". As Dodd \& Sweeney (2009) I also think that Corazza et al. (2002) fail to criticize the purity status of the indexical "I". Finally, I have presented examples that criticize the logical truth status of "I am here now" in virtual environments. In both cases it is clear that at least "here" is not a pure indexical. It is also showed that there is a sense in which we can say that "I" refers to an avatar, but it is not a problem since, by definition, the avatar always represents the person behind the computer. Following Putnam (1973) I think that the extension of our terms depends upon the actual nature of the particular things that serve as paradigms. Semantic theories must encompass contributions of society and, by examining the social nature of virtual environments, it's plausible [in some cases] to shift the use of the indexical word "here" to refer to a virtual space where avatars interact, where "I" [using an avatar to represent me] am "here" [in a VE] "now" [at the moment the sentence is tokened].

\section{References}

Brinck, I. (1997). The Indexical "I”. London: Kluver Academic Publishers, Synthese Library.

Colterjohn, J., \& MacIntosh, D. (1987). Gerald Vision and Indexicals. Analysis, 47, 58-60. http://dx.doi.org/10.1093/analys/47.1.58

Corazza, E., Fish, W., \& Gorvett, J. (2002). Who Is I? Philosophical Studies, 107, 1-21. http://dx.doi.org/10.1023/A:1013111419036

Dodd, D., \& Sweeney, P. (2009). Indexicals and Utterance Production. Philosophical Studies (forthcoming).

Kaplan, D. (1989). Demonstratives. In J. Almog, J. Perry, \& H. Wettstein (Eds.), Themes from Kaplan (pp. 481-565). Oxford: Oxford University Press.

Ludlow, P. (2009). Virtual Communities, Virtual Cultures, Virtual Governance. In The 2009 Compass Interdisciplinary Virtual Conference. New Jersey: Wiley-Blackwell. http://compassconference.wordpress.com/about/conference-program/

Ondrejka, C. (2007). Second Life: Collapsing Geography. Innovations, 2, 27-54. http://dx.doi.org/10.1162/itgg.2007.2.3.27

Perry, J. (1997). Indexicals and Demonstratives. In R. Hale, \& C. Wright (Eds.), Companion to the Philosophy of Language (pp. 586-612). Oxford: B. Blackwell.

Perry, J. (2001). Reference and Reflexivity. Stanford, CA: CSLI Publications.

Perry, J. (2003). Predelli's Threatening Note: Contexts, Utterances and Token in the Philosophy of Language. Journal of Pragmatics, 35, 373-387.

Predelli, S. (1998a). I am not Here Now. Analysis, 58, 107-115.

Predelli, S. (1998b). Utterance, Interpretation, and the Logic of Indexicals. Mind and Language, 13, 400-414.

${ }^{8}$ Avatars range from lifelike humans to more fanciful robots, animals, and mythical creatures. 
Predelli, S. (2005). Contexts. Meaning, Truth, and the Use of Language. New York: Oxford University Press.

Putnam, H. (1973). Meaning and Reference. The Journal of Philosophy, 70, 699-711. http://dx.doi.org/10.2307/2025079

Romdenh-Romluc, K. (2002). Now the French Are Invading England. Analysis, 62, 34-41. http://dx.doi.org/10.1093/analys/62.1.34

Sidelle, A. (1991). The Answering Machine Paradox. Canadian Journal of Philosophy, 81, 525-539.

Zalta, E. (1988). Logical and Analytic Truths That Are Not Necessary. The Journal of Philosophy, 85, 57-74. http://dx.doi.org/10.2307/2026992 